\title{
Ordinal position in the serial learning of rats
}

\author{
RICHARD A. BURNS, JOHN A. DUNKMAN, JR., and STACY L. DETLOFF \\ Southeast Missouri State University, Cape Girardeau, Missouri
}

\begin{abstract}
Rats were runway trained on each of two, three-trial series consisting of different varieties of reward $(\mathrm{X}, \mathrm{Y}$, and $\mathrm{Z}$ ) and nonreward (N) serving as trial outcomes. The two series are represented as XNY and ZNN. Distinguishing the two series were different brightness and texture cues on the runway floor. Transfer tests, conducted after the rats had developed faster running for rewarded trials than for nonrewarded trials and slower running on Trial 2 of ZNN than on Trial 2 of XNY, provided evidence that trial position, rather than item memories, was controlling the discriminations. In Experiment 1, reversing the floor cues completely reversed the discriminations. In Experiment 2, transfer to NNN did not change the routine patterns of approach that had been established.
\end{abstract}

After nearly a century of interpreting the evidence on serial learning in humans from the perspective of theories that emphasized the interconnections or associations among the items in a serial list, theoretical efforts shifted (Bower, 1971; Ebenholtz, 1972). The new effort featured not the items themselves but the positions they occupy in the list. That information about the ordinal position of a list item may be a basis for serial learning came primarily from experiments examining transfer to lists in which originallist items occupied either the same position or different positions (Ebenholtz, 1972).

As was the case in the study of serial learning with humans, appreciation for the role played by position information in animal learning trailed an initial theoretical effort that focused attention on the interitem associations animals might form when routinely confronted with consistent sequences (e.g., Capaldi, 1967, 1994; Capaldi \& Molina, 1979). In one example of a serial procedure with animals, rats were trained on a pair of three-trial series of rewards in a runway (Burns, Wiley, \& Payne, 1986). The series were distinguished by differing trial outcomes, one series ending with a rewarded trial $(\mathrm{R})$, the other ending with nonreward $(\mathrm{N})$. Only the initial trials of the series were the same; the first was always rewarded, the second was not, so the series may be represented RNR and RNN. The runway floor, which could be made smooth and black or rough and white, was used as a condition to differentiate the two series. In this experiment, and in others like it (e.g., Burns, Wiley, \& Stephens, 1986; Capaldi, Nawrocki, \& Verry, 1983), a couple of interesting results occurred. The rats learned to approach the goal on all trials of both series in a manner that was generally in accord with the

This research was supported by a Grants and Research Funding grant from Southeast Missouri State University. The authors thank H. D. Kimmel for his valued counsel on earlier versions of this manuscript. Correspondence concerning this work should be addressed to the first author, Department of Psychology, Southeast Missouri State University, One University Plaza, Cape Girardeau, MO 63701 (e-mail: rburns@semovm.semo.edu). reward outcome: They ran faster to rewards than to nonrewards. Their approach was slower on Trial 2, however, if Trial 3 was not rewarded (i.e., slower on Trial 2 of RNN than on Trial 2 of RNR).

The theoretical analysis offered by Burns, Wiley, and Payne (1986) for this pair of findings stressed the learning of the ordinal position for the items in the series. Sources of stimulation correlated with item position were considered to be conditioned to running by the reward outcomes on the various trials, reward producing stronger conditioning than nonreward. Thus, the animals come to run faster on the first trial position than on the second and either fast or slow on the third trial position, conditional on the floor (series) cue. The trial position cues for the second and third positions in the series are not completely unique, and generalization may occur. For this reason, behavior on Trial 2 may resemble behavior on Trial 3, and the animals may run slower in the second position of RNN than in the same position of RNR. That the degree of distinctiveness of the position cues was the determining factor in the Trial 2 differences on the RNR and RNN series was suggested by the results of an intentional increase in position distinctiveness. This increase was accomplished by inserting a unique long interval between the second and third trials of the two series, replacing the short interval that typically separates the trials in these experiments. So, in the Burns, Wiley, and Payne investigation, the second trial position was preceded by a $30-\mathrm{sec}$ interval, whereas the third position was preceded by a 10 -min interval. Making the trial positions distinct eliminated the differences in approach on the second trials of the two series.

An alternative analysis of the typical findings with these procedures (Capaldi et al., 1983) made use of the initial theoretical emphasis in animal serial learning, and it has apparent intuitive appeal as well. By this interitem account, the memorial representations of specific reward or nonreward outcomes become interconnected forming a "serial map," so that experience with any item represented in the map would retrieve representations not only of that item but also of the other items in the list. These repre- 
sentations were the presumed basis for the animal's anticipation of both current and remote events in the series. Slower approach on the second trial of the RNN series than on the same trial of the RNR series was viewed as interevent, or remote, anticipation, which is supposed to make rats run slower when anticipating a future nonreward than when anticipating a future reward. They would also run slower anticipating a current nonreward than a current reward.

Of course, this interitem account of serial learning does not need to be viewed as incompatible with the position account. The treatment of the problem offered by Capaldi, Alptekin, Miller, and Birmingham (1997) stresses that position and interitem cues are independent sources of information that can each become learned. Nevertheless, Capaldi et al. (1997) regard item memories the predominant source of information. The position analysis offered by Burns, Wiley, and Payne (1986) characterizes item memories as one source of information about position in typical serial procedures. When serial learning procedures do not confound trial position and item memories, item memory or position may function independently.

Separating the contribution of memory from other position cues has been difficult, given that, in most serial learning investigations with animals, item memories and the other position cues are confounded. Capaldi and Miller (1988b) have described an RNR/RNN study that attempted to show item memories are the basis of serial learning in rats by making those memories the only reliable source of information distinguishing the two series. In previous investigations, the conditional cue that distinguished the two series was either a floor cue (e.g., Burns, Wiley, \& Payne, 1986) or the ordinal position of the series itself, either first or second in every training day (Burns, Wiley, \& Stephens, 1986; Capaldi et al., 1983). Capaldi and Miller (1988b) used no cue other than the kind of reward administered on the first trials of the different series. Using three kinds of rewards, Noyes pellets and different breakfast cereals, the series were labeled XNY and ZNN, in which $\mathrm{X}, \mathrm{Y}$, and $\mathrm{Z}$ designated the different rewards. Capaldi and Miller (1988b) found that rats approached both rewarded and nonrewarded trials correctly and approached Trial 2 of ZNN more slowly than Trial 2 of XNY. These results showed that item memories could serve effectively as conditional series cues.

Evidence for the efficacy of item memories as series cues, however, is not necessarily evidence that item memory, independent of serial position, was the basis of these reward outcome discriminations and of what Capaldi and Miller (1988b) called interevent anticipation.

\section{EXPERIMENT 1}

Experiment 1 examined the $\mathrm{XNY} / \mathrm{ZNN}$ procedure when two conditional series cues were intentionally confounded. Rats were trained on XNY and ZNN, for which
$X$ was Noyes pellets and $Z$ and $Y$ were units of distinctively flavored commercial cereals. The different first-trial rewards of the series provide a possible item-memory cue to differentiate the two series, but an additional series cue was contributed by the floor of the runway. A rough and white floor, for instance, was always in effect during the XNY series, and a smooth and black floor was always in effect during ZNN (relation of floor and series was counterbalanced). Both interitem and position accounts predict the usual pattern of results: differentiated running on rewarded and nonrewarded trials with running slower still on Trial 2 in ZNN than on the same trial in XNY. To separate theoretical predictions, a cue-reversal transfer test was arranged on Day 31. If a particular floor cue was a condition of the XNY (or ZNN) series during training, it was for a single test day the condition of ZNN (or XNY).

The reversal of the floor cues allows us to determine whether or not item memories are the predominant source of information not only for differentiating the series but also for differentiating trials, or positions, within the series. Interitem theory predicts that the reversal of floor cues would have one of two consequences: (1) To the extent that item memories, as series cues, prevail over the floor cues, there should be no change in the pattern of approach during the transfer test. (2) If item memories are not the predominant series cue, but those memories combine with the conditional floor cue to form the basis of approach within the series, the reversal should produce a general disruption and loss of reward-outcome discriminations. This disruption should occur because the new combination of memory and floor cues would produce conflicting representations of trial sequences.

Position theory predicts that the floor-cue reversal would reverse the pattern of approach. Animals would run on XNY as if it were ZNN, and they would run on ZNN as if it were XNY. This result should occur because the combination of floor cue and trial position would not be broken by the reversal. The only disruption expected by position theory would be slight because the item memories, which would be changed in the test, are but one of many sources of information about position.

\section{Method}

Subjects. The subjects were 6 experimentally naive male albino rats obtained from the Holtzman Company, Madison, WI. The rats were about 90 days old at the beginning of preliminary training.

Apparatus. The apparatus was an L-shaped wooden runway, $233.7 \mathrm{~cm}$ long and $10.0 \mathrm{~cm}$ wide, covered with vented clear plastic. Measures of total running times were taken from a silent digital clock that was activated when the manually operated start door was opened and stopped when the rats crossed a photobeam mounted $195.6 \mathrm{~cm}$ beyond the start door. Entry into the goal area was initiated by a right turn, $182.9 \mathrm{~cm}$ beyond the door, that led to an aluminum goal cup, $7.62 \mathrm{~cm}$ in diameter and $4.45 \mathrm{~cm}$ deep. The entire runway was painted black, but a floor panel made of aluminum truckbumper stock with raised diamond patterns was painted white and hinge-mounted so that the floor of the runway could easily be made rough and white or smooth and black. 


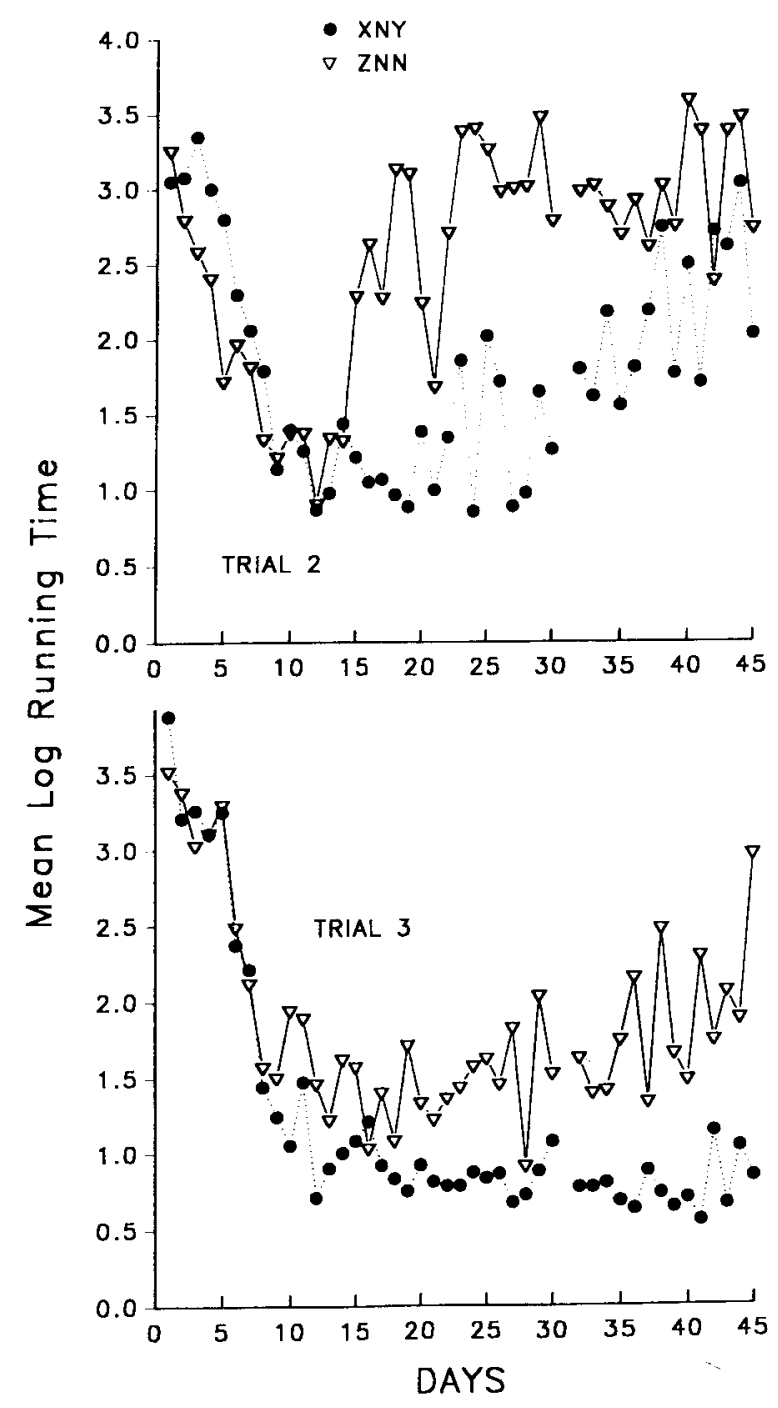

Figure 1. Mean transformed running times on Trial 2 (top panel) and Trial 3 (bottom panel) of both XNY and ZNN series over the 45 days of training in Experiment 1. The figure does not include a point for Day 31, the day on which the floor cues were reversed.

Procedure. After arrival in the laboratory, the rats were assigned to individual living cages and maintained for 3 days with ad-libitum food, Purina Rodent Chow, and water. They were allowed at least $1 \mathrm{~h}$ per day in a group exercise box, where they were handled periodically. After the initial period in the lab, and for the remainder of the experiment, the rats were allowed free access to water and $16 \mathrm{~g}$ of food each day following their training and a period of at least $15 \mathrm{~min}$ in the group box. The 4th day began a series of runway exploration days, the 1 st of which consisted of placing the rats individually in the runway for $5 \mathrm{~min}$, with the start and retrace doors being opened and closed several times at about $2.5 \mathrm{~min}$ into the exploration. On the following day, the exploration included six $0.045-\mathrm{g}$ Noyes pellets (X) scattered throughout the runway. In 2 subsequent exploration days, six units of distinctively flavored commercial breakfast cereals were in the runway, Captain Chocos (Y) on the 1st day and Apple Jacks $(Z)$ on the 2 nd day. This procedure was re- peated once for each reward type. The order of running of the rats during this exploration phase and during all subsequent training phases was determined randomly each day. For a random half of the rats, the $X$ and $Y$ exploration days were conducted in the runway with the smooth black floor, and the $Z$ days were with a rough white floor. For the remaining rats, $X$ and $Y$ were with the rough white floor and $Z$ was with the smooth black floor.

During the first phase of primary training, each rat received two, three-trial series ( $X N Y$ and $Z N N$ ) each day for 30 experimental days. A trial began with the opening of the start door about $2 \mathrm{sec}$ after the rat was placed in the start area, and it ended when the rat had consumed the reward (4 pellets or 1 unit of cereal) or after $30 \mathrm{sec}$ in the unbaited goal on nonrewarded $(\mathrm{N})$ trials. The order in which the series were presented varied randomly over days, and the floor cues associated with XNY and $Z N N$ were the same for each rat, counterbalanced, as those in exploration training. Each rat ran all three trials of a given series in succession, a procedure that produces an interval between trials of $15-30 \mathrm{sec}$. Because the second series of the day was begun only after all rats had completed the first series, the interval between series was $15-30 \mathrm{~min}$. In the beginning of this training, only one series was run each day, so that 4 calendar days were required to complete the first 2 experimental days.

A transfer test was conducted on Day 31. On this single test day, the floor cues associated with the two series were reversed for all rats. For the rats whose training conditions involved XNY and ZNN occurring in smooth black and rough white runways, respectively, the transfer test had XNY appearing for the first time in a rough white runway and $\mathrm{ZNN}$ in a smooth black runway. An equivalent reversal was made for the remaining rats. All procedures, except the floor reversal, during the test were exactly as they had been during the original training.

Conditions were returned to those of original training for Days 32-36; on Day 37, a second transfer test was administered. The second test involved reversing the reward type on the first trials of the two series making the effective series ZNY and XNN, with the floor cues for ZNY being the same as they had been for XNY and those for XNN being the same as for the previous $\mathrm{ZNN}$.

The experiment was completed after a final return to the original training conditions for Days 38-45.

\section{Results}

All running times were subjected to a normalizing transformation $[x=\log (x)]$ for analysis. Figure 1 is a plot of the average transformed running times to the different reward outcomes in the second and third trial positions. The figure shows two curves, one for the XNY series and one for ZNN, and it does not include points for the Day 31 reversal test.

A repeated measures analysis of variance (ANOVA), conducted over the first 30 days, evaluated the factors of days, series, and trials. It was coupled with subsequent analyses on each individual trial position. The differences among trials were significant $[F(2,10)=79.71, p<.01]$. Running was significantly faster on the first trial than on any other, and it was slower on the second trial than on the third $[L S D(10)=.199, p<.05]$. Further evaluation of the reliable trials $\times$ series interaction $[F(2,10)=8.95, p<$ $.01]$ showed that the rats approached the goal on the first trial in a way that did not depend on whether the series was $\mathrm{XNY}(M=0.90, S E=0.07)$ or $\mathrm{ZNN}(M=0.89, S E=0.04)$ $(F<1)$. The second trial position, however, produced clear differences $[F(1,5)=20.66, p<.01]$, with approach 


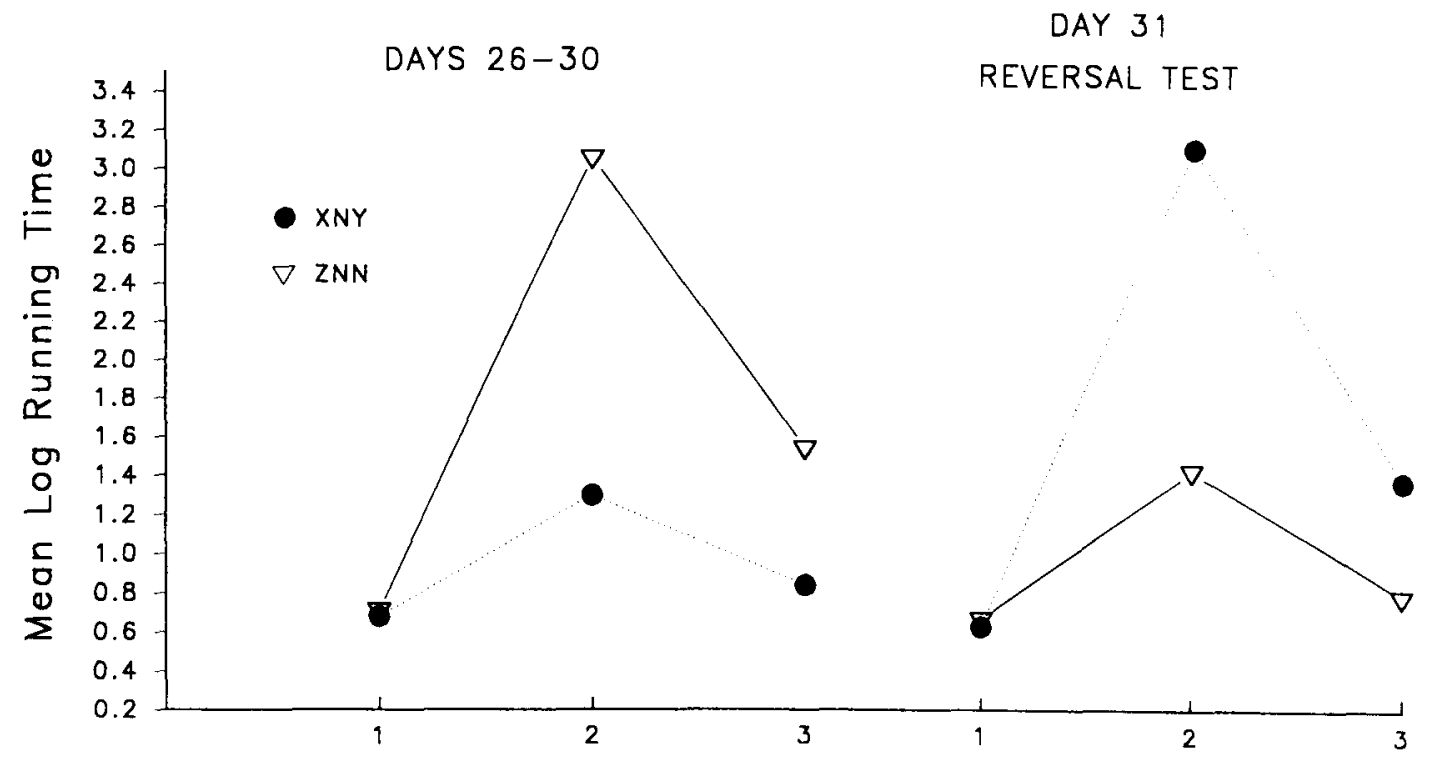

TRIALS

Figure 2. Mean transformed running times for each of the trial positions for XNY and ZNN on the training days (left panel) preceding the reversal test (right panel) conducted in Experiment 1.

on that trial of XNY being significantly more rapid ( $M=$ $1.62, S E=0.08)$ than on the same trial of $Z N N(M=2.37$, $S E=0.09)$. Differences in the same direction were evident on the third trial as well $[F(1,5)=7.51, p<.05]$, where approach was again faster on XNY $(M=1.44, S E=0.08)$ than on ZNN $(M=1.84, S E=0.08)$.

The effects of reversing the conditional floor cues while maintaining the relevance of the item-memory cues are depicted in Figure 2, which shows performance on the three trial positions just prior to the reversal (Days 26-30) and on the test day (Day 31). The rats approached the three elements of the XNY series on the reversal test just as they had approached ZNN before the test. Approach to ZNN when the series cue was reversed was as it had been to $\mathrm{XNY}$ before the reversal. On the test day, the trials $\times$ series interaction was again significant $[F(2,10)=5.83, p<$ $.05]$. The rats ran significantly slower $[L S D(25)=.456$, $p<.05]$ on the second trial of the XNY series $(M=3.12$, $S E=0.25)$ than on any other trial, and they ran slower on the third trial of the XNY series $(M=1.38, S E=0.40)$ than on the same trial of the ZNN series $(M=0.79, S E=0.15)$.

The absence of any disruption when item-memory cues on the first trial of the two series were reversed on Day 37 is evident in Figure 1. The series discrimination on the third trial during this final training period appeared to have improved, whereas those differences on the second trial, while maintained, appeared to diminish. As was the case before the Day 31 reversal, trials was a significant factor $[F(2,10)=50.82, p<.01]$ during Days 32-45, with faster running on the first trial $[L S D(10)=.426$, $p<.05]$ and slower running on the second than on either of the other trials. There was an interaction of trials $\times$ series $[F(2,10)=13.01, p<.01]$, which, when examined, showed that running was equally fast on the first trials of $\mathrm{XNY}$ and $\mathrm{ZNN}[F(1,5)=1.83, p>.05]$ but differentially slow on the second trials $[F(1,5)=14.80, p<.05]$. During this period, the rats approached the goal more slowly on the second trial of ZNN $(M=3.00, S E=0.10)$ than on the second trial of XNY $(M=2.16, S E=0.12)$. Robust differences $[F(1,5)=34.73, p<.01]$ were observed on the third trial, where the rats approached nonreward $(M=$ $1.86, S E=0.14)$ more slowly than reward $(M=0.77, S E=$ $0.04)$.

\section{Discussion}

The theoretical implications of the cue-reversal results are clear. In this procedure, item memories of the particular reward outcomes did not control the discriminations established during the initial training phase. Reversing the conditional series cue caused the rats to approach the XNY series as if it were $\mathrm{ZNN}$ and caused the rats to approach the ZNN series as if it were XNY. If the specific memories of $X$ and $Z$ were the effective series cues and were also the basis of reward discriminations within the series, the reversal of the floor cues should not have led to reversal of XNY and ZNN behavior. Capaldi and Miller (1988b) concluded that item memories were the probable dominant series cues for a group of 4 rats trained with both memory and series order as possible series cues, because they found no significant differences between that 
group and the group described earlier, which had only memory cues. Reversing their series order may have fostered a different conclusion.

If the floor cues, rather than item memories, had become the functional series cues in the present investigation and were conditionally combined only with item memories to produce the reward discriminations within the series, a general disruption and loss of reward discriminations should have occurred during the reversal. But this is not what occurred. The reversal of item-memory cues on Day 37 again failed to produce disruption, a result that invites the same difficulties as the reversal findings for any view that conceptualizes item memories as predominant and independent of position cues in this procedure.

These results favor a position interpretation. If information about the ordinal position of each trial in the series is conditioned to approach by the reward outcome on each trial, the rats should develop a routine pattern of approach on the XNY series that is fast for the first trial position, slow for the second, and fast for the third. The routine would be fast-slow-slow for ZNN. Once these routines are established, temporary changes in the particular items within the series should not alter the approach pattern if the integrity of the three trial positions is maintained, as it was in these transfer tests.

The differences observed on the second trial position of the XNY and ZNN series are interpreted as being the result of generalization between the position cues associated with the second and third positions. Position cues on the first trial are assumed to be relatively unique. Less discriminable are the position cues for the second and third trial positions. If position cues for the third position of the XNY series partially generalize to those of the second position, approach on the second position should be more rapid than on the same trial of the ZNN series. This, of course, is a typical result, and it was the result observed. As training continues, the degree of generalization among the trial positions should decrease, resulting in clearer Trial 3 differences and diminishing Trial 2 differences, an expectation supported by the findings of Experiment 1 .

Generalization caused by similar position cues may occur from trial to trial (position to position) within a series, and it may also occur from one series to another, more from the same trial of a different series than from a different trial. For this reason, running on the second trial of ZNN should be slower than running on the third trial of the same series, as it typically is and was in Experiment 1. On Trial 2, generalization from the same trial in the XNY series would make approach to that trial of ZNN slower, and similar generalization on Trial 3 would make approach faster than on Trial 2 of ZNN. This faster running on Trial 3 than on Trial 2 of ZNN is also a typical result that does not seem to be predicted by interitem theory unless interevent anticipation of the first trial of an upcoming series is assumed. If, however, anticipation can encompass several series, we would expect it also to encompass all three trials within a series. But, faster running times on Trial 1 of XNY than on Trial 1 of ZNN have not been observed.

\section{EXPERIMENT 2}

The particular rewards used in Experiment 1, Noyes pellets and flavored breakfast cereals, have been employed in several other empirical investigations, with good evidence that they provide easily discriminable item memories. In studies designed to examine numerical competence in rats, Burns and Gordon (1988), Burns, Goettl, and Burt (1995), and Capaldi and Miller (1988a) used pellets and cereals to examine the rat's ability to categorize numbers of trials depending on the type of reward represented in those categories. Of course, Capaldi and Miller (1988b) used these rewards to show that the specific memories of $\mathrm{X}$ and $\mathrm{Z}$ in the $\mathrm{XNY} / \mathrm{ZNN}$ procedure could be employed by rats as an effective series cue. There is ample evidence that pellets and cereals provide distinctive item memories.

In spite of that evidence, a failure of pellet and cereal rewards to contribute distinctive memories in Experiment 1 may have been responsible for the findings in the cue-reversal shift. The interitem theory does not require that the rewards provide distinctive memories, except, as in the Capaldi and Miller (1988b) investigation, when those particular reward memories were necessary to distinguish one series from another. The item memory could be merely of reward rather than of a particular reward type. If, for instance, the floor cues overshadowed the particular item-memory cues, the effective series in Experiment 1 would have been RNR and RNN.

An early experiment by Wike and King (1973) examined three-trial series in the runway using nonreward, a single 0.045 -g Noyes pellet, and a $0.500-\mathrm{g}$ pellet. One group received a uniformly increasing magnitude of reward over the three trials, whereas another received a uniformly decreasing magnitude over trials. After performance stabilized, a 12-day extinction period was conducted using the same three-trial procedure as in original training, but without rewards. Wike and King found that the pattern of running established during training on the three-trial series persisted well into extinction. This is a result that would not be expected if the pattern in training was due to the development of a serial map based on interitem associations. A similar result was obtained by Burns, Hulbert, and Cribb (1990).

In Experiment 2, transfer tests were administered from original training with $\mathrm{XNY}$ and $\mathrm{ZNN}$ to series in which nonreward was the only outcome on every series trial (NNN). Position theory predicts that performance in the NNN test will be as it was prior to the shift because the integrity of the positions in the three-trial series is maintained in the shift. Interitem theory predicts disruption because the memory of nonreward, the only available item memory in the transfer test, was an event linked to upcoming nonrewards and rewards, whether specific or 


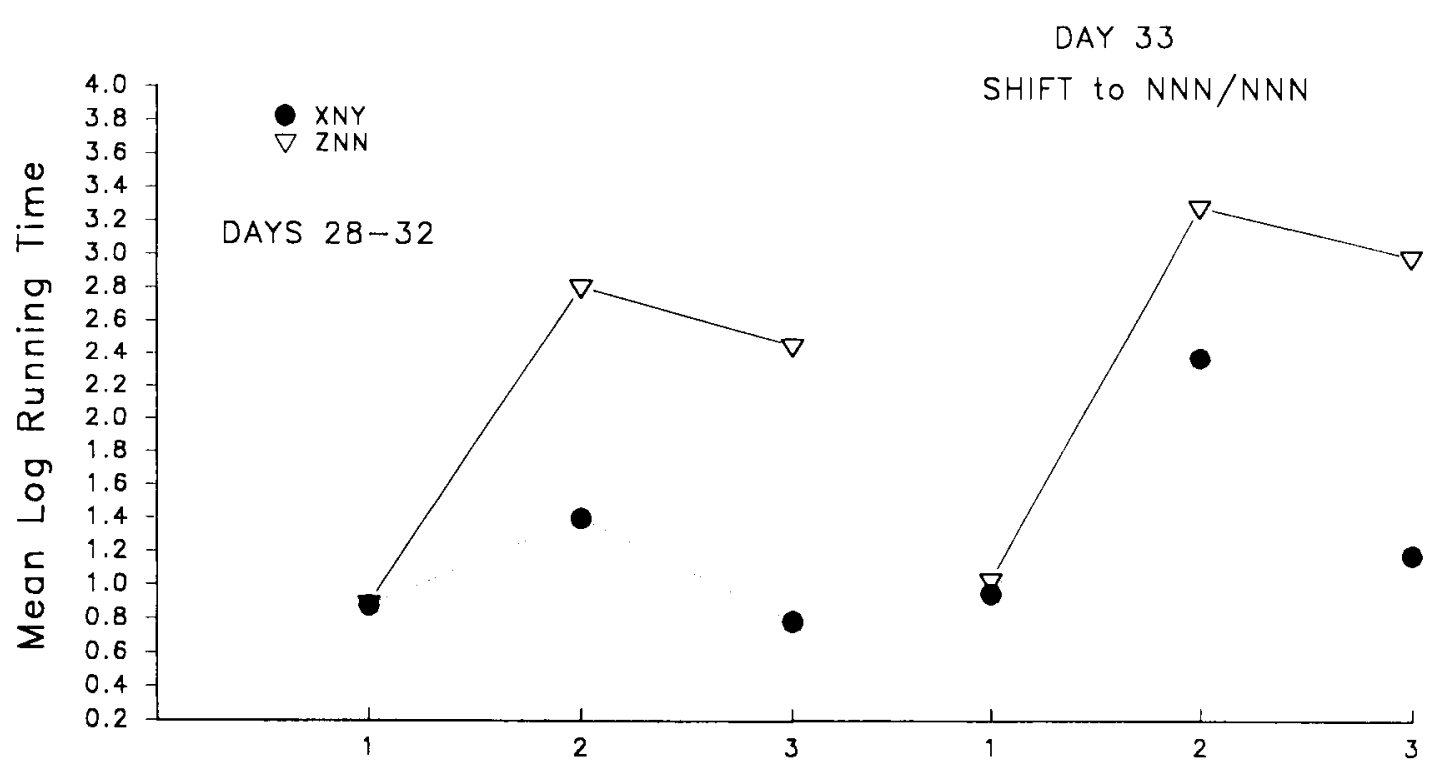

\section{TRIALS}

Figure 3. Mean transformed running times in Experiment 2 for each of the XNY and ZNN trial positions at the end of original training (left panel) and on the day during which transfer to series containing only nonreward was examined (right panel).

general, as well as to previous specific or general rewards in the serial map.

\section{Method}

Subjects and Apparatus. The subjects were 6 naive rats of the same description as those used in Experiment 1. The runway was the same runway described in Experiment 1.

Procedure. The procedures for preliminary treatment, food rationing, group exercise, and handling were essentially the same as those of Experiment 1. Runway exploration was conducted as before, except that different cereals were used, CoCo Roos (Y) and King Vitaman $(Z)$. Noyes pellets $(0.045 \mathrm{~g})$ again served as reward on the initial trial of the XNY series. The rats were given runway exploration with the various rewards scattered throughout the runway on different exploration days, and the counterbalanced floor cues that were appropriate for each reward type were used.

Primary training lasted 32 days and was conducted in the same manner as in Experiment 1, with the rats being run in random orders determined each day and with relatively short intervals between trials within the XNY and ZNN series but longer intervals separating the series. The order of the series, first or second in each training day, was also determined randomly, so that the series cues were the brightness and texture of the floor and the specific item memories of $X$ and $Z$.

On Day 33, the procedures were the same as during original training, except that all trials in both series terminated in nonreward, a $30-\mathrm{sec}$ confinement in the goal area with no reward in the cup.

\section{Results}

The running times were transformed logarithmically and are plotted in Figure 3, showing the average times for each of the three trial positions on both daily series. The left panel shows the times for the final 5 days of original training with $\mathrm{XNY} / \mathrm{ZNN}$, and the right panel shows times for the day on which the series were NNN/NNN.

A repeated measures ANOVA covering Days 28-32 showed that the trials $\times$ series interaction was significant $[F(2,10)=10.65, p<.01]$. Follow-up tests $[\operatorname{LSD}(105)=$ $.318, p<.05]$ showed that the rats ran equally fast on the first trials of the series and slower on the second trial of the ZNN series $(M=2.81, S E=0.16)$ than on any other trial of either series. They approached the goal faster on the third trial of XNY $(M=0.79, S E=0.04)$ than on the same trial of ZNN $(M=2.46, S E=0.21)$. The same sort of analysis of the findings for the day in which all trials were nonrewarded produced the same pattern of results. When further evaluated, the significant trials $\times$ series interaction $[F(2,10)=15.65, p<.01]$ showed no differences in the running times on the first trials of the two series, which were faster $[L S D(25)=.731, p<.05]$ than on any other trials except the third trial of what had been the XNY series. On the second trial of the series that had been $\mathrm{XNY}$, running times were faster $(M=2.38, S E=0.44)$ than on the same trial of the series that had been $Z \mathrm{NN}$ $(M=3.29, S E=0.37)$. Times on the final trial differed as well, with faster approach on the previous XNY series $(M=1.18, S E=0.18)$ than on the previous $\mathrm{ZNN}(M=2.99$, $S E=0.41)$.

\section{Discussion}

In Experiment 2, the routine pattern of approach that was established during primary training was maintained in the transfer test even though the only item memory re- 
trieved during the test would have been the memory of nonreward. It is unclear what the exact nature of the disruption caused by experiencing nonreward should be, according to interitem theory, on the series of trials in the NNN shift. There is no reason from either theoretical perspective to think that the first trials of the shift would be approached any differently than they had been before the shift, because the rat would not yet have experienced the changed reward values that interitem theory predicts would cause disruption. Position theory predicts routine approach to the cues associated with the first trial position, no matter what the reward outcome. Whether the changes, once experienced, would invoke only the remaining elements of a serial map- $\mathrm{N}$, or whatever the second $\mathrm{N}$ elicits, for $\mathrm{ZNN}$, and $\mathrm{Y}$ for $\mathrm{XNY}$ - even though there was no experience in the shift with the initial components $(Z$ or $X)$ is an interitem issue that has not been examined. It is also not clear whether interitem theory predicts a specific kind of disruption following the second $\mathrm{N}$ on the second trial of the NNN shift. This issue arises because, although experience with $\mathrm{N}$ and $\mathrm{NN}$ had happened in original training, and, therefore, those elements were components of the serial maps that were theoretically formed, we cannot tell whether the map that is instated by the experience should be unaffected by the absence of the usual previous components of the map.

If it is assumed that changed previous elements of the serial map would have no effect at all on the reinstatement of only the remaining elements, the rats, according to interitem theory, should have run equally slowly on the second and third trials of the NNN series that occurred with the prevailing floor cue associated with ZNN (or RNN if $Z$ had never been distinguished from $X$ and $Y$ ). This prediction is based on an assumption that the first $\mathrm{N}$ in the NNN series elicits the subsequent second $\mathrm{N}$ in the $\mathrm{ZNN}$ map. If the rat treats the first $N$ in the NNN series as the second $\mathrm{N}$ in the $\mathrm{ZNN}$ map, we might expect this memory to elicit the map of XNY, the only events that followed the second $N$ of $Z N N$ (or $R N N$ ) in original training. A reinstated map of XNY would presumably cause the rat to run fast on the second and third trials of NNN. They should have run fast as well on all three trials of what had been XNY (or RNR).

The findings of Experiment 2 instead recommend a position interpretation. The routine approach to the three trial positions established in training should have been maintained during the shift, even if the particular elements of the series appeared in different positions, because the cues associated with position were not changed in the shift (Ebenholtz, 1972). To the extent that item memory is itself a position cue, one of several, there may have been some minor disruption in the shift, but there was not.

\section{GENERAL DISCUSSION}

The results of Experiments 1 and 2 produce a very different conclusion from that reached by Capaldi and Miller (1988b) in a study similar to the present research but with- out the transfer tests. Capaldi and Miller (1988b) reasoned correctly that their demonstration of reward discriminations and "interevent anticipation" in the XNY/ZNN procedure, when only the memories of $X$ and $Z$ were available to distinguish the two series, must have meant that item memories were the effective series cues. We agree with that conclusion. Furthermore, there is ample evidence (see, e.g., Capaldi, 1994) that rats use memory from previous rewards as cues in a wide variety of other learning procedures. The disparity in our interpretations stems from further conclusions reached by Capaldi and Miller (1988b), who reasoned that the efficacy of the memories as a series cue meant that position cues could not have been contributing to the discriminations they examined. Because the XNY and ZNN series they studied were presented irregularly, position of the series could not have been the effective series cue; but Capaldi and Miller (1988b) concluded from this that the remote anticipation on Trial 2 and the correct current anticipation of both $\mathrm{Y}$ and $\mathrm{N}$ on Trial 3 could not be explained by position cues.

It is important to understand that series cues and cues associated with the position of trials within a series are different cues. Neither the position theory nor the interitem theory predicts reward differentiation on the third trials of XNY and ZNN if an effective series cue had not been provided. From the position theory, however, any reliable cues may serve as series cues in these procedures, combining with cues linked to the ordinal position of trials within a series and forming the basis of the discriminations. Among many others that might combine with position, the series cues could be the brightness and texture of a runway, the ordinal position of the series itself, or the specific memories of different Trial 1 outcomes. That the memory of the Trial 1 outcome was an effective series cue in the Capaldi and Miller (1988b) investigation does not in itself mean that position cues are not involved in the Trial 2 and Trial 3 discriminations.

In the study of serial learning in people, a theoretical emphasis on position learning eventually received strong support (e.g., Bower, 1981), but interest in position has been only sporadic in the study of animals (e.g., Chen, Swartz, \& Terrace, 1997; Couvillon, Brandon, Woodard, \& Bitterman, 1980; D'Amato \& Colombo, 1988; Roitblat, Pologe, \& Scopatz, 1983; Straub \& Terrace, 1981). For example, Roitblat et al. (1983) found that replacing individual reward magnitudes with nonreward in a welllearned five-trial series of differing magnitudes did not affect rats' established patterns of approach. A recent experiment with rhesus monkeys (Chen et al., 1997) employed a derived-list procedure in which position intergrity was either maintained or disrupted. When position was maintained, learning of a derived list was immediate.

We view the available evidence as a justification for taking more seriously the role of position learning in the general theoretical analysis of serial learning and numerical processes in animals. To argue for increased attention to position learning is not to argue against interitem 
memories. It is to argue for a theoretical approach that integrates position and item information.

Due in part to lack of attention to the position question, the nature of position cues is improperly understood. Burns, Wiley, and Payne (1986) suggested that position cues could arise from temporal intervals beginning at series outset, the number of trials previously encountered in a series, inevitable (but unintentional) trial-to-trial variations in experimental procedure, and intentional trial-totrial variations, such as those examined by Burns, Wiley, and Payne (1986) and Burns, Wiley, and Stevens (1986). Any source of stimulation, including specific item memories, that is correlated with the ordinal position of a trial could be employed as position cues, and most serial learning procedures include these cues.

The analysis of position learning offered here relies on what might be thought of as traditional constructs in animal learning theory. It is essentially an S-R reinforcement theory (Hull, 1943) that views position stimuli as being conditioned to behavior by reward. The assumptions about generalization are borrowed from an earlier literature (e.g., Spence, 1936), just as were many of the assumptions of Bower (1971) and Ebenholtz (1972) in the human learning tradition. The view that generalization and discrimination are intimately related empirically and theoretically is also in the tradition (Brown, 1961), and this view may be used as a traditional alternative to describe behavior that appears to others as evidence of remote anticipation of imminent events (Capaldi, 1994).

Concern with position learning in animals has, however, found voices that reflect the contemporary cognitive orientation (e.g., D'Amato, 1991; D'Amato \& Colombo, 1988; Roitblat et al., 1983). Encouraged by apparent comparative differences in associative transitivity experiments, D'Amato and his colleagues (reviewed in D'Amato, 1991) have proposed that, in monkeys (Cebus apella), a lucid internal representation of the series elements and their positions is formed, and the animal accesses this representation and scans it from initial to final positions. This theoretical orientation bears some similarity to the serial map of Capaldi et al. (1983), but it emphasizes position information rather than item information. Whatever theoretical orientation turns out to be correct, the evidence we report and review here seems to require theory to include the role played by position information, and it counsels an approach that does not consider interitem associations a process that is independent of position learning.

\section{REFERENCES}

Bower, G. H. (1971). Adaptation-level coding of stimuli and serial position effects. In M. H. Appley (Ed.), Adaptation-level theory. New York: Academic Press.

Bower, G. H. (1981). Theories of learning. Englewood Cliffs, NJ: Prentice-Hall.

Brown, J. S. (1961). The motivation of behavior. New York: McGrawHill.

Burns, R. A., GoettL, M. E., \& Burt, S. T. (1995). Numerical dis- criminations with arrythmic serial presentations. Psychological Record, 45, 95-104.

Burns, R. A., \& Gordon, W. U. (1988). Some further observations on serial enumeration and categorical flexibility. Animal Learning \& Behavior, 16, 425-428.

Burns, R. A., Hulbert, L. G., \& CribB, D. (1990). A test for order relevance in a three-element serial learning task. Journal of General Psychology, 117, 91-98.

Burns, R. A., Wiley, L. P., \& Payne, T. L. (1986). Temporal cuing of runs in series of reward events reduces interevent anticipation. Animal Learning \& Behavior, 14, 190-196.

Burns, R. A., Wiley, L. P., \& Stevens, J. (1986). Interevent anticipation with external cuing of runs and sucrose rewards. Psychological Record, 36, 101-107.

CAPALDI, E. J. (1967). A sequential hypothesis of instrumental learning. In K. W. Spence \& J. T. Spence (Eds.), The psychology of learning and motivation (Vol. 1, pp. 67-156). New York: Academic Press.

CAPAlDI, E. J. (1994). The sequential view: From rapidly fading stimulus traces to the organization of memory and the abstract concept of number. Psychonomic Bulletin \& Review, 1, 156-181.

Capaldi, E. J., Al.ptekin, S., Miller, D. J., \& Birmingham, K. M. (1997). Is discriminative responding in reward outcome serial learning mediated by item memories of position cues? Learning \& Motivation, 28, 153-169.

CaPAldi, E. J., \& Miller, D. J. (1988a). Counting in rats: Its functional significance and the independent cognitive processes that constitute it. Journal of Experimental Psychology: Animal Behavior Processes, 14, 3-17.

CAPAldi, E. J., \& Miller, D. J. (1988b). The rat's simultaneous anticipation of remote events and current events can be sustained by event memories alone. Animal Learning \& Behavior, 16, 1-7.

Capaldi, E. J., \& Molina, P. (1979). Element discriminability as a determinant of serial-pattern learning. Animal Learning \& Behavior, 7. 318-322.

Capaldi, E. J., Nawrocki, T. M., \& Verry, D. R. (1983). The nature of anticipation: An inter- and intraevent process. Animal Learning \& Behavior, 11, 193-198.

Chen, S., Swartz, K. B., \& Terrace, H. S. (1997). Knowledge of ordinal position of list items in rhesus monkeys. Psychological Science. 8, 80-86.

Couvillon, P. A., Brandon, S., Woodard, W. T., \& Bitterman, M. E. (1980). Performance of pigeons in patterned sequences of rewarded and nonrewarded trials. Journal of Experimental Psychology: Animal Behavior Processes, 6, 137-154.

D'Amato, M. R. (1991). Comparative cognition: Processing of serial order and serial pattern. In L. Dachowski \& C. F. Flaherty (Eds.), Current topics in rat learning: Brain, emotion, and cognition (pp. $165-$ 185). Hillsdale, NJ: Erlbaum.

D'Amato, M. R., \& Colombo, M. (1988). Representation of serial order in monkeys (Cebus apella). Journal of Experimental Psychology: Animal Behavior Processes, 14, 131-139.

EBENHoltz, S. M. (1972). Serial learning and dimensional organization. In G. H. Bower (Ed.), The psychology of learning and motivation (Vol. 5, pp. 267-314). New York: Academic Press.

Hull, C. L. (1943). Principles of behavior. New York: Appleton-CenturyCrofts.

Roitblat, H. L., Pologe, B., \& Scopatz, R. A. (1983). The representation of items in serial position. Animal Learning \& Behavior, 11. 489-498.

SPENCE, K. W. (1936). The nature of discrimination learning in rats. Psychological Review, 43, 427-449.

Straub, R. O., \& Terrace, H. S. (1981). Generalization of serial lcarning in the pigeon. Animal Learning \& Behavior, 9, 454-468.

WIKE, E. L., \& KING, D. D. (1973). Sequences of reward magnitude and runway performance. Animal Learning \& Behavior, 1, 175-178.

(Manuscript received October 1, 1998; revision accepted for publication February 17, 1999.) 